\title{
Análise da qualidade energética da falha de pinhão para a produção de briquetes
}

\section{Analysis of the energy quality pinion fails to produce briquettes}

\author{
Rodolfo Cardoso Jacinto', Martha Andreia Brand ${ }^{2}$, Polliana D'angelo Rios², \\ Alexsandro Bayestorff da Cunha ${ }^{2}$ e Gabriel Allegretti ${ }^{3}$
}

\begin{abstract}
Resumo
A inclusão do uso energético das falhas de pinhão na cadeia produtiva do pinhão, na serra catarinense, pode incrementar a renda na agricultura familiar, contribuindo para a melhoria na qualidade de vida dos agricultores. Desta forma, o objetivo deste trabalho foi determinar a qualidade energética das falhas do pinhão in natura e dos briquetes produzidos a partir destes resíduos. Foram utilizadas falhas do estróbilo feminino (pinhas) de árvores de Araucaria angustifolia (Bert.) O. Ktze, coletadas em três municípios da região serrana sul do estado de Santa Catarina, sendo estes: Lages, Painel e São José do Cerrito. Foram avaliadas as propriedades físicas, energéticas, químicas e a granulometria das falhas e, após a produção dos briquetes em briquetadeira piloto de laboratório, também suas propriedades físicas e energéticas. A umidade foi de alta para as falhas in natura $(61 \%)$, inviabilizando seu uso energético sem prévio tratamento. Já para os briquetes foi de $9,91 \%$. O teor de extrativos em água quente $(9,84 \%)$; álcool tolueno $(13,18 \%)$ e lignina $(47,76 \%)$ foram altos, tornando o material apto à produção de compactados de alta qualidade. A qualidade energética das falhas de pinhão é diferente da madeira (valores médios de espécies madeireiras dos grupos das Gimnospermas e Angiospermas) e similar ao bagaço de cana e ramas de algodão. $O$ teor de carbono fixo $(22,44 \%)$ e cinzas $(4,26 \%)$ para as falhas foram maiores que na madeira e o poder calorífico superior (4208 kcal. $\mathrm{kg}^{-1}$ para falha e $4443 \mathrm{kcal} . \mathrm{kg}^{-1}$ para os briquetes) foi menor que na madeira. A massa específica aparente dos briquetes foi de $1146 \mathrm{~kg} \cdot \mathrm{m}^{-3}$. Não foi necessário moer as falhas para a produção de briquetes. A produção de briquetes a partir de falhas de pinhão pode ser uma alternativa viável de uso dos resíduos da cadeia produtiva do pinhão, gerando renda para pequenos produtores rurais.
\end{abstract}

Palavras-chave: compactados; biomassa, resíduos, escamas da araucária.

\begin{abstract}
The inclusion of the use of seed-empty strobili for energy purposes in Araucaria angustifolia production chain, in Santa Catarina, can increase income in family agriculture, helping to improve the quality of life of farmers. Thus, the objective of this study was to determine the energy quality of these failures in nature and briquettes produced from their wastes. Female strobili with seed-set failures were used from Araucaria angustifolia (Bert.) O. Ktze, collected in three municipalities in the southern mountain region of the state of Santa Catarina: Lages, Painel and São José do Cerrito. The physical, energy, chemical and particle size of those failed strobili were determined. After production of briquettes in a pilot briquetteer, the physical and energetic properties were also determined in briquettes. Moisture content on wet base was high for failures in nature $(61 \%)$, preventing its energy use without prior treatment, and low in the briquettes $(9.91 \%)$. The extractives in hot water $(9.84 \%)$; toluene alcohol $(13.18 \%)$ and lignin $(47.76 \%)$ were high, making the materials suitable for production of high quality compressed briquettes. The energy quality of seed devoid strobili (failures) is different from wood (average values of timber species groups of Gymnosperms and Angiosperms) and similar to sugar cane bagasse and cotton branches. The fixed carbon content $(22.44 \%)$ and ash $(4.26 \%)$ for the failures were higher than for the wood and the gross calorific value $\left(4208 \mathrm{kcal}^{\mathrm{kg}} \mathrm{kg}^{-1}\right.$ for failure and $4443 \mathrm{kcal}^{\mathrm{kg}}{ }^{-1}$ for the briquettes) was lower than that for wood. The apparent density of the briquettes was $1146 \mathrm{~kg} \cdot \mathrm{m}^{-3}$. No grinding of failures for the production of high quality briquettes is required. The production of briquettes from failed seed-set strobili can be a viable alternative use of waste of strobili production chain, generating income for small farmers.
\end{abstract}

Keywords: compacted; biomass, waste, sterile scales of araucaria

\footnotetext{
${ }^{1}$ Mestrando do Programa de Pós-graduação em Engenharia Florestal. UDESC - Universidade do Estado de Santa Catarina. Av. Madre Bencenuta, 2007 - Itacorubi, Florianópolis, SC. E-mail: rodolfo_cj@hotmail.com.

${ }^{2}$ Professor do Departamento de Engenharia Florestal. UDESC - Universidade do Estado de Santa Catarina. Av. Madre Bencenuta, 2007 - Itacorubi, Florianópolis, SC. E-mail: martha.brand@udesc.br; polliana.rios@udesc.br; alexsandro.cunha@udesc.br.

${ }^{3}$ Engenhario Florestal. UDESC - Universidade do Estado de Santa Catarina. Av. Madre Bencenuta, 2007 - Itacorubi, Florianópolis, SC. E-mail: rodolfo cj@hotmail.com.
}

Sci. For., Piracicaba, v. 44, n. 112, p. 821-829, dez. 2016 DOI: dx.doi.org/10.18671/scifor.v44n112.04 


\section{INTRODUÇÃO}

A Araucaria angustifolia (Bertoloni) Otto Kuntze., árvore característica da vegetação do sul do Brasil, já teve grande importância econômica nos ciclos de madeira da década de 1940 e 1960, sendo gradativamente substituída por espécies do gênero Pinus para usos da madeira na forma sólida e para a produção de papel de fibra longa.

As utilizações mais comuns da araucária estão associadas à obtenção de madeira para tabuados, vigamentos, pranchões, caixas, móveis; cabos de vassoura e ferramentas, palitos de dente e de fósforo, fabricação de compensados, pasta mecânica e celulose, papel, instrumentos musicais, instrumentos de adorno, artigos de esporte, tacos de nós, mourões, telhas de tábuas. Os galhos e refugos, e, especialmente, o nó de pinho, servem para lenha e combustível de caldeiras, e os pinhões servem de alimento para o homem e para os animais (GUERRA et al, 2002). Além de ser utilizada também para arborização urbana e paisagismo em casas, jardins e praças (VIEIRA-DA-SILVA et al, 2011). No entanto, uma das utilizações mais nobres da espécie é para alimentação, a partir do consumo dos seus pinhões; sendo esta iguaria muito apreciada na culinária do sul do Brasil, conforme mencionam alguns autores como Santos (1973) e Vieira (2004).

Atualmente, o uso madeireiro desta espécie é limitado pela disponibilidade de outras fontes de matéria-prima e restrições legais. No entanto, tem sido incentivado e estudado com maior intensidade o uso da semente desta árvore, principalmente na alimentação humana, inclusive por participar das atividades tradicionais culinárias das regiões de ocorrência da espécie e por seu apelo turístico. As iniciativas políticas, econômicas e científicas são fortemente voltadas para a agricultura familiar, enfocando a cadeia produtiva do pinhão como renda complementar para famílias de pequenos agricultores.

No entanto, o pinhão é apenas um dos componentes do estróbilo feminino da araucária (pinha). A pinha tem quatro componentes: sementes (pinhão), eixo central, escamas estéreis ou não fertilizadas (falhas) e escamas férteis não fertilizadas ou abortadas (pinhões chochos). Uma pinha tem seu peso distribuído em média entre 44 - 55\% de pinhão, de 3 - 9\% do eixo central, de 1 10\% de pinhões chochos e de 48 - 54\% de "falhas" (MANTOVANI et al. 2004; VIEIRA-DA-SILVA; REIS, 2009).

Segundo Vieira-da-Silva et al. (2011), nas regiões de São Francisco de Paula, RS e Painel, SC, as "falhas" da araucária possuem um potencial para serem utilizadas em práticas de base agroecológica e também para confecção de artesanatos a partir de produtos naturais. Os usos detectados pelos autores foram: adubo; matéria seca para composteira; cobertura de solo, para diminuição de capina e para a não utilização de herbicidas; para confecção de artesanato e para tingimento natural de lãs.

Tais utilizações elucidam práticas alternativas que podem ser inseridas dentro do manejo agroecológico das regiões de Floresta Ombrófila Mista (Floresta com araucária), e otimizando um recurso já coletado. Portanto, a utilização das falhas de pinhão pode aumentar a renda para o agricultor que coleta pinhão, pois este terá 2 produtos que podem ser comercializados. O primeiro, o pinhão, que já possui um canal de comercialização consolidado. E o segundo a "falha", este com um canal de comercialização que está sendo construído, mas que também pode ser utilizada dentro da própria propriedade (VIEIRA-DA-SILVA et al, 2011).

Considerando ainda que as regiões de ocorrência natural da araucária possuem clima temperado com invernos rigorosos, mais uma alternativa de uso das falhas de pinhão seria sua aplicação energética, como um combustível alternativo para sistemas de aquecimento residencial ou comercial.

Em termos de qualidade energética das falhas do pinhão, Oliveira et al (2010) constataram que o teor de umidade da falha do pinhão recém produzida foi de $64 \%$, com densidade aparente de 0 , $916 \mathrm{~g} . \mathrm{cm}^{-3}$, considerando este teor de umidade. No teste de granulometria, 98,74\% do material ficou

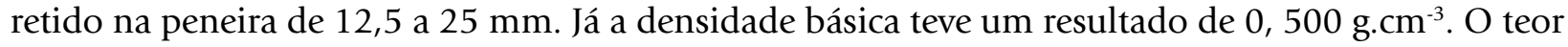
de cinzas foi de 3,54\%. O poder calorífico superior (PCS) teve um resultado de $4810 \mathrm{kcal}^{\mathrm{kg}}{ }^{-1}$, poder calorífico inferior (PCI) de $4486 \mathrm{kcal} \mathrm{kg}^{-1}$ e poder calorífico líquido (PCL) de $1367 \mathrm{kcal}^{\mathrm{kg}}{ }^{-1}$. Estes autores demonstraram que as falhas possuem um poder calorífico similar ao da madeira do Pinus, que segundo Brand (2010) varia de 4500 a $4800 \mathrm{kcal} \mathrm{kg}^{-1}$, tornando o material apto à geração de energia.

Esta análise energética das falhas já dá um indicativo da qualidade do material para se tornar insumo energético num processo de geração de energia. O teor de umidade é alto, demonstrando que 
o uso direto em processos térmicos de geração de energia não é eficiente, portanto a compactação pode ser uma forma de melhoria da qualidade energética deste resíduo. Os testes granulométricos indicam que o material é homogêneo, característica importante para a compactação e favorável para as falhas. A densidade é média, permitindo boa compactação do material. O teor de cinzas é baixo e compatível com outros tipos de biomassa como a madeira, por exemplo. O poder calorífico é similar a outras fontes de biomassa, como a madeira e outros resíduos agrícolas mencionados por Brand (2010). O poder calorífico líquido é baixo, podendo ser melhorado a partir da redução do teor de umidade.

Desta forma, a determinação de formas de uso das falhas do pinhão terá impactos positivos tanto sociais, pela geração de empregos na atividade de uso do pinhão; econômicos, pelo aumento da renda para os agricultores familiares na região Sul do Brasil e ambientais pela utilização de um resíduo que de outra forma ficaria disposto no ambiente, acarretando em passivos ambientais.

Atualmente, o aumento da importância do uso da biomassa para geração de energia abre a possibilidade de análise de materiais alternativos, principalmente os resíduos de processos produtivos para a geração de energia. Isso torna as falhas do pinhão um material potencial para estudos nesta área.

Assim, o objetivo deste trabalho foi determinar a qualidade energética das falhas do pinhão e dos briquetes produzidos a partir deste resíduo.

\section{MATERIAL E MÉTODOS}

Os materiais utilizados para o estudo foram as falhas de pinhão obtidas a partir do estróbilo feminino (pinhas) de árvores de Araucaria angustifolia, coletadas em três municípios da região serrana sul do Estado de Santa Catarina: Lages (Amostra 1), Painel (Amostra 2) e São José do Cerrito (Amostra 3). Foram coletadas as falhas que já haviam sido separadas das pinhas pelos agricultores que comercializam o pinhão. Após a coleta, as amostras foram embaladas em pacotes fechados, para que não houvesse perda de umidade e encaminhados para processamento e análise.

As propriedades analisadas nas falhas de pinhão in natura e nos briquetes podem ser visualizadas na Tabela1, juntamente com as normas utilizadas para os ensaios.

Tabela 1. Propriedades físicas, químicas e energéticas analisadas nas falhas de pinhão in natura e nos briquetes.

Table 1. Physical, chemical and energy properties analyzed on the seed-empty strobili in natura and the briquettes.

\begin{tabular}{|c|c|c|}
\hline Material analisado & Analise realizada & Norma \\
\hline $\begin{array}{l}\text { Falhas inteiras e moídas } \\
\text { Briquetes }\end{array}$ & Teor de umidade na base úmida & NBR 14929 (ABNT, 2003b) \\
\hline \multirow{3}{*}{ Falhas moídas } & Solubil.em água & NBR 14577(ABNT, 2003a) \\
\hline & Solubil.em álcool tolueno & NBR 14853 (ABNT, 2010a) \\
\hline & Lignina insolúvel em ácido & NBR 7989 (ABNT, 2010b) \\
\hline \multirow{2}{*}{$\begin{array}{l}\text { Falhas moídas } \\
\text { Briquetes }\end{array}$} & Análise imediata (teor de cinzas, carbono fixo e voláteis) & ASTM D1762 (ASTM, 2007) \\
\hline & Poder calorífico superior e líquido & DIN 51900 (DIN, 2000). \\
\hline Briquetes & Massa específica aparente & Método estequiométrico \\
\hline
\end{tabular}

A análise imediata foi realizada em termobalança gravimétrica (TGA), com temperaturas de 900 ${ }^{\circ} \mathrm{C}$ para a determinação dos voláteis e $700{ }^{\circ} \mathrm{C}$ para cinzas. O poder calorífico superior foi determinado em calorímetro. A partir do poder calorífico superior determinado no calorímetro foram calculados o poder calorífico inferior e líquido, com base na composição química elementar do combustível (porcentagem de hidrogênio) e dos teores de umidade e de cinzas determinados em laboratório previamente.

As amostras passaram pelo teste de granulometria, utilizando-se um conjunto de peneiras com abertura de tela de: $6,3 \mathrm{~mm}, 4,75 \mathrm{~mm}, 2 \mathrm{~mm}, 0,85 \mathrm{~mm}$ e uma base e um agitador eletrônico de peneiras, com agitação por 15 minutos. A determinação da granulometria foi realizada através da equação 1 :

Onde: $\mathrm{G}=$ Granulometria em \%;

$$
G=\frac{P_{1}\left(P_{2}, P_{3} \ldots P_{n}\right)}{\sum P} x 100
$$

$P_{1^{\prime}} P_{2^{\prime}} P_{3^{\prime}} P_{4}=$ Peso da amostra em cada peneira em gramas;

$\Sigma \mathrm{P}=$ Somatório do peso das amostras; 
Uma briquetadeira laboratorial hidráulica à quente foi utilizada para a produção de 18 briquetes constituídos de falhas inteiras ( 9 briquetes) e de falhas moídas ( 9 briquetes). A quantidade de amostra para os briquetes moídos foi de $50 \mathrm{~g}$, e para os briquetes de falha inteira foi de $30 \mathrm{~g}$. Para a confecção dos briquetes, tanto as falhas inteiras com as moídas estavam com o teor de umidade de equilíbrio de $12 \%$.

A temperatura de ensaio foi de $120^{\circ} \mathrm{C}$, a pressão utilizada foi de $5 \mathrm{MPa}$ por 10 minutos. Este procedimento foi desenvolvido para simular as condições reais de briquetadeiras industriais.

Após esse tempo a pressão foi aumentada para $13 \mathrm{MPa}$ por 2 minutos, seguida da liberação gradual da pressão após o término do ensaio e manutenção do corpo-prova sob compressão suave até seu resfriamento. As propriedades energéticas e físicas determinadas nos briquetes podem ser visualizadas na Tabela 1.

Todos os dados obtidos foram submetidos à análise estatística, utilizando ANOVA/MANOVA e o teste de Tukey, com o programa StatSoft.

\section{RESULTADOS E DISCUSSÃO}

\section{Qualidade das falhas de pinhão}

A Tabela 2 mostra as propriedades físicas e energéticas das falhas de pinhão das três amostras analisadas no estudo.

Tabela 2. Propriedades físicas e energéticas da falha de pinhão.

Table 2. Physical and energetic properties of seed-empty strobili.

\begin{tabular}{llcccccc}
\hline Amostra & TU (\%) & PCS (kcal.kg-1) & PCL (kcal.kg $\left.\mathbf{~}^{-1}\right)$ & TV (\%) & CF (\%) & TC (\%) \\
\hline 1 & 62 a & 4144 a & 972 c & 74,98 a & 20,17 b & 4,85 a \\
2 & $57 \mathrm{c}$ & 4259 a & 1262 a & $71,58 \mathrm{c}$ & 23,64 a & 4,77 a \\
3 & $61 \mathrm{~b}$ & $4223 \mathrm{a}$ & $1085 \mathrm{~b}$ & $73,33 \mathrm{~b}$ & $23,50 \mathrm{a}$ & $3,17 \mathrm{~b}$ \\
Média & 61 & 4208 & 1106 & 73,30 & 22,44 & 4,26 \\
\hline
\end{tabular}

Nota: TU: Teor de umidade na base úmida; PCS: Poder calorífico superior; PCL: Poder calorífico líquido no teor de umidade apresentado na coluna TU; TV: Teor de voláteis; CF: Teor de carbono fixo; TC: Teor de cinzas. Amostra I: Procedência Lages; Amostra 2: Procedência Painel; Amostra 3: Procedência São José do Cerrito. Médias seguidas da mesma letra, na coluna, não apresentam variação estatística significativa para o Teste de Tukey $(\mathrm{P}>0,05)$.

O teor de umidade das amostras analisadas foi alto, mesmo considerando que os equipamentos mais modernos planejados para a queima de partículas úmidas possam operar com teor de umidade máximo de 60\%, na prática os valores máximos considerados são 50\% (BRAND, 2010). No entanto, para a geração de energia, quanto menor a umidade, melhor será a eficiência energética do combustível.

Houve variação estatística significativa entre todas as amostras, que pode ser explicada pelo fato da coleta ter sido feita em falhas que haviam sido removidas das pinhas previamente, com diferentes tempos de estocagem nos produtores, antes da análise.

$\mathrm{O}$ alto teor de umidade observado indica que o uso direto das falhas de pinhão, sem prévia secagem ou tratamento é inviável, pois acarretará em baixo poder calorífico líquido, como pode ser observado na Tabela 2. Sistemas de geração e co-geração (termelétricas) que utilizam biomassa florestal para a geração de energia tem como padrão o valor mínimo de $1900 \mathrm{kcal} \mathrm{kg}^{-1}$ como mencionam Furtado et al. (2012).

O poder calorífico superior das falhas foi menor que o valor apresentado por Oliveira et al (2010) para falha de pinhão, e menor comparativamente a outros tipos de biomassa, incluindo a madeira, como pode ser visualizado na Tabela 3. O valor mais próximo ao poder calorífico superior das falhas de pinhão são as ramas de algodão e o bagaço de cana.

O poder calorífico é um excelente parâmetro para se avaliar a potencialidade energética de combustíveis de biomassa (PROTÁSIO et al, 2011; BRAND, 2010; FRIEDLET et al., 2005; PARIKH et al, 2005), sendo definido como a quantidade de energia liberada na combustão completa de uma unidade de massa do material combustível e apresenta significativa dependência da constituição química elementar e mineral da biomassa (PROTÁSIO et al., 2011).

De forma geral, entre as amostras analisadas não houve diferença estatística para o poder calorífico superior, sendo que para o poder calorífico líquido as diferenças entre as amostras foi inversa à variação do teor de umidade, o que já era esperado, visto que o teor de umidade é utilizado no cálculo do poder calorífico líquido. 
Quanto à composição química imediata das falhas de pinhão, tanto a porcentagem de carbono fixo, como voláteis e teor de cinzas se assemelham ao resíduo denominado ramas de algodão, e o teor de voláteis também é similar ao bagaço de cana, porém este último tem maior teor de cinzas, como pode ser visualizado na Tabela 3.

Comparativamente à madeira, as falhas de pinhão possuem maior porcentagem de carbono fixo e menor de voláteis. O teor de cinzas em geral é baixo, porém maior que na madeira. Segundo Obernberger e Thek (2002) e Werther et al. (2000) combustíveis com alto índice de carbono fixo tem queima mais lenta, implicando maior tempo de residência destes dentro dos queimadores, em comparação com outros que tenham menor teor de carbono fixo.

Tabela 3. Composição química imediata de diferentes tipos de biomassa a base $0 \%$ de teor de umidade.

Table 3. Immediate chemical composition of different types of biomass the basis of $0 \%$ moisture content.

\begin{tabular}{lccc|c}
\hline \multirow{2}{*}{ Tipo de biomassa } & \multicolumn{3}{c|}{ Composição imediata média (\%) } & PCS \\
\cline { 2 - 5 } & TV (\%) & TC (\%) & CF (\%) & kcal.kg-1 \\
\hline Madeira de Coníferas & 82,54 & 0,29 & 17,7 & 4700 \\
Madeira de Folhosas & 81,42 & 0,79 & 17,82 & 4600 \\
Casca de arroz & $65,47-67,8$ & $17,89-18,6$ & $13,6-16,67$ & $3700-3850$ \\
Bagaço de cana & 73,78 & 11,27 & 14,95 & $3700-4100$ \\
Casca de coco & 67,95 & 8,25 & 23,8 & 4500 \\
Sabugos de milho & 80,1 & 1,36 & 18,54 & 4500 \\
Ramas de algodão & 73,29 & 5,51 & 21,2 & 4370 \\
Cama de aviário & 62,73 & 23,4 & 13,87 & 3550 \\
Capim elefante & 76,69 & 5,6 & 17,7 & 3600 \\
\hline
\end{tabular}

Fonte: Brand, 2010.

Nota: TV: Teor de voláteis; CF: Teor de carbono fixo; TC: Teor de cinzas; PCS: Poder calorífico superior.

Houve diferença estatística entre todas as amostras para o teor de voláteis. Para carbono fixo a Amostra 1 das demais e para cinzas a Amostra 3 das outras.

A distribuição granulométrica das falhas de pinhão está apresentada na Tabela 4 .

Tabela 4. Distribuição granulométrica das partículas de falha de pinhão moída em moinho de martelo.

Table 4. Particle size distribution of seed-empty strobili produced in hammer mill.

\begin{tabular}{lccccc}
\hline Amostra & Acima de 6,3 $\mathbf{~ m m}$ & $\mathbf{4 , 7 5} \mathbf{~} \mathbf{m}$ a $\mathbf{6 , 2 9} \mathbf{~} \mathbf{~ m}$ & $\mathbf{2} \mathbf{~} \mathbf{m m}$ a 4,74 $\mathbf{~ m m}$ & $\mathbf{0 , 8 5} \mathbf{~} \mathbf{m}$ a 1,99 $\mathbf{~} \mathbf{m m}$ & Abaixo de 0,84 $\mathbf{~ m m}$ \\
\hline 1 & $6,49 \mathrm{a}$ & $8,17 \mathrm{~b}$ & $22,57 \mathrm{a}$ & $37,40 \mathrm{a}$ & $25,36 \mathrm{a}$ \\
2 & $9,65 \mathrm{a}$ & $11,45 \mathrm{a}$ & $24,04 \mathrm{a}$ & $28,75 \mathrm{~b}$ & $26,10 \mathrm{a}$ \\
3 & $9,51 \mathrm{a}$ & $12,88 \mathrm{a}$ & $22,92 \mathrm{a}$ & $24,24 \mathrm{c}$ & $30,44 \mathrm{a}$ \\
\hline Média & 8,62 & 10,83 & 23,18 & 30,13 & 27,30 \\
\hline
\end{tabular}

As amostras apresentaram proporções diferentes de finos, que são as partículas menores que 4,75 mm. Para a Amostra 1 os finos compuseram 85,33\% da amostra. Já para amostra 2 e 3, a quantidade de finos foi de 78,99\% e 77,6\%, respectivamente. Segundo Protásio et. al. (2011), amostras com menores granulometrias podem influenciar a densidade aparente da biomassa e dos briquetes produzidos.

A granulometria da falha do pinhão está abaixo do recomendado por Dias et al. (2012), que menciona que a biomassa destinada à compactação deve ser reduzida para 6 a $8 \mathrm{~mm}$ de tamanho, podendo conter cerca de $10-20 \%$ de finos, exceto para materiais que possuem pequeno tamanho de partículas, como serragem e casca de arroz.

Segundo Kaliyan e Morey (2009), o tamanho da partícula é influenciador importante da durabilidade e resistência dos briquetes, evidenciando a importância da classificação granulométrica da biomassa antes da compactação.

O tamanho de partículas é muito importante para determinar a qualidade e durabilidade de um pellet ou briquete. Quanto menor o tamanho de partícula, menor será a porosidade do produto final e portanto, maior será sua densidade. A baixa porosidade do pellet ou briquete pode, entretanto dificultar a queima devido ao menor número de espaço livre para a difusão de calor. Sendo assim, a taxa de combustão dos produtos muito densos será menor e maior o período de combustão (DIAS et al., 2012). 
Segundo Dias et al. (2012), estudos mostram que a condição ideal é a presença de partículas de diferentes tamanho, pois isso possibilita o melhor empacotamento das partículas e contribui para a alta resistência dos briquetes e pellets.

As propriedades químicas das falhas de pinhão podem ser visualizadas na Tabela 5.

Tabela 5. Propriedades químicas da falha de pinhão.

Table 5. Chemical Properties of seed-empty strobili.

\begin{tabular}{lccc}
\hline Amostra & Lignina (\%) & Solubilidade em água fria (\%) & Solubilidade em álcool tolueno (\%) \\
\hline 1 & $48,88 \mathrm{a}$ & $9,60 \mathrm{a}$ & $8,44 \mathrm{~b}$ \\
2 & $45,15 \mathrm{~b}$ & $10,33 \mathrm{a}$ & $17,85 \mathrm{a}$ \\
3 & $49,25 \mathrm{a}$ & $9,59 \mathrm{a}$ & $13,26 \mathrm{ab}$ \\
Média & 47,76 & 9,84 & 13,18 \\
\hline
\end{tabular}

Nota: Médias seguidas da mesma letra não apresentam variação estatística significativa para o Teste de Tukey $(P>0,05)$.

A lignina, as proteínas, amidos, gorduras e carboidratos solúveis, são adesivos naturais da biomassa. A presença desses compostos justifica a não utilização de aglomerantes artificiais para a briquetagem ou a peletização (DIAS et al., 2012). A lignina atua como um agente ligante in situ no material. As temperaturas elevadas amolecem e ajudam no processo de ligação (KALIYAN; MOREY, 2009). Entretanto, caso a matéria-prima tenha deficiência desses compostos, há necessidade da adição do ligante (DIAS et al., 2012).

Segundo Kaliyan e Morey (2009), devido à aplicação de alta pressão e temperatura, pontes sólidas podem ser desenvolvidas por difusão de moléculas a partir de uma partícula para o outro ponto de contato. Pontes sólidas podem também ser formadas entre as partículas devido à cristalização de alguns ingredientes, reações químicas, o endurecimento das pastas, e solidificação dos componentes derretidos. Principalmente, após o resfriamento, finas camadas de adsorção são imóveis e podem formar ligações fortes entre as partículas adjacentes, que por alisar a rugosidade da superfície e aumenta a área de contato entre partículas ou diminuia distância entre partículas e permite que as forças atrativas intermoleculares participem no mecanismo de ligação.

Sob alta pressão, os componentes de ligação, proteínas, lignina e pectina nas matérias-primas ou da biomassa são espremidos para fora das partículas, isso contribui para a ligação entre partículas (KALIYAN; MOREY, 2009).

Os valores de lignina obtidos para a falha de pinhão são maiores que os observados para espécies madeireiras do grupo das Gimnospermas, como as do gênero Pinus. Neste contexto, pode ser destacado o estudo realizado por Balloni (2009), que determinou o valor de $28 \%$ de lignina para Pinus elliotii. Moraes et al. (2005), trabalhando com Pinus oocarpa, encontrou o valor médio de 25,18\% de lignina. Já Brito e Nuci (1984) encontraram o valor de 31\% para Pinus oocarpa; 31,8\% de lignina para $P$. caribea var. hondurensis e $33,6 \%$ de lignina para $P$. caribea var. bahamensis. Vale ressaltar ainda que quanto maior a quantidade de lignina, melhor será a ligação interna nos briquetes.

Os valores de extrativos em água e álcool tolueno também foram maiores que os observados para madeira. Este comportamento era esperado visto que as falhas se constituem em parte protetora das sementes, nos estróbilos femininos da araucária.

Brito e Nuci (1984) encontraram valores de 5,3\% e Moraes et al. (2005) encontraram 4,38\% de extrativos em etanol:tolueno, para Pinus oocarpa. Brito e Nuci (1984) encontraram o valor de 5,1\%, tanto para $P$. caribea var.hondurensis, quanto para $P$. caribea var. bahamensis de extrativos em etanol:tolueno.

Portanto, as propriedades químicas das falhas de pinhão tornam esta matéria-prima potencial e apta à produção de compactados para a geração de energia.

\section{Qualidade dos briquetes de falhas de pinhão}

Os valores de teor de umidade, massa específica aparente, poder calorífico e análise imediata dos briquetes produzidos a partir das falhas de pinhão são apresentados na Tabela 6 .

O teor de umidade dos briquetes foi baixo, havendo variação significativa entre os tratamentos quando comparados de forma conjunta, mas não quando a comparação foi feita entre briquetes de falhas inteiras e moídas. Segundo Kaliyan e Morey (2009), em geral, o teor de umidade final dos briquetes deve ser inferior a $13 \%$. 
Tabela 6. Propriedades físicas e energéticas dos briquetes de falhas de pinhão.

Table 6. Physical and energetic properties of the seed-empty strobili briquettes.

\begin{tabular}{|c|c|c|c|c|c|c|c|}
\hline Amostra & TU (\%) & MEA $\left(\mathbf{k g} \cdot \mathrm{m}^{-3}\right)$ & PCS (kcal.kg-1) & PCL (kcal.kg $\left.{ }^{-1}\right)$ & TV (\%) & CF (\%) & TC (\%) \\
\hline FI 1 & $10,55 \mathrm{~b}$ & $1130 \mathrm{a}$ & $4508 a b$ & $3679 a$ & $72,32 \mathrm{a}$ & $24,79 a$ & $2,89 \mathrm{~b}$ \\
\hline $\mathrm{FI} 2$ & $10,10 \mathrm{~b}$ & $1148 \mathrm{a}$ & 4577 a & 3763 a & $72,15 \mathrm{a}$ & $24,81 \mathrm{a}$ & $3,05 \mathrm{~b}$ \\
\hline $\mathrm{FI} 3$ & $8,45 \mathrm{~cd}$ & $1166 \mathrm{a}$ & 4566 a & 3833 a & $72,35 a$ & $24,99 a$ & $2,66 \mathrm{~b}$ \\
\hline FM 1 & $9,63 \mathrm{bc}$ & $1100 \mathrm{a}$ & 4440 ac & 3662 a & $71,92 \mathrm{a}$ & $24,31 \mathrm{a}$ & $3,77 \mathrm{a}$ \\
\hline FM 2 & $7,80 \mathrm{~d}$ & $1164 \mathrm{a}$ & 4309 bc & 3627 a & $72,84 \mathrm{a}$ & $24,50 \mathrm{a}$ & $2,66 \mathrm{~b}$ \\
\hline FM 3 & $12,90 \mathrm{a}$ & $1169 a$ & $4257 \mathrm{bc}$ & $3348 b$ & - & - & - \\
\hline Média & 9,91 & 1146 & 4443 & 3652 & 72,32 & 24,68 & 3,00 \\
\hline $\mathrm{FI}$ & $9,70 \mathrm{a}$ & $1148 \mathrm{a}$ & 4550 a & 3758 a & $72,23 \mathrm{a}$ & $24,80 \mathrm{a}$ & $2,97 \mathrm{a}$ \\
\hline $\mathrm{FM}$ & 9,76 a & $1144 \mathrm{a}$ & $4335 b$ & $3546 \mathrm{~b}$ & $72,38 \mathrm{a}$ & $24,41 \mathrm{a}$ & $3,22 \mathrm{a}$ \\
\hline
\end{tabular}

A massa específica aparente dos briquetes foi alta. Protásio et al. (2011), analisando briquetes de diferentes matérias-primas obteve massa específica aparente de $946 \mathrm{~kg} \cdot \mathrm{m}^{-3}$ para briquetes de serragem de eucalipto; $934 \mathrm{~kg} . \mathrm{m}^{-3}$ para briquetes de resíduo de milho e $1291 \mathrm{~kg} . \mathrm{m}^{-3}$ para briquetes de casca de café. Furtado et al. (2010) analisando briquetes feitos com diferentes misturas de resíduos de Pinus obtiveram densidades aparentes de $1390 \mathrm{~kg} \cdot \mathrm{m}^{-3}$ para briquetes de casca; $1220 \mathrm{~kg} \cdot \mathrm{m}^{-3} \mathrm{para}$ briquetes de cavacos; $1240 \mathrm{~kg} \cdot \mathrm{m}^{-3}$ para briquetes de serragem e $1210 \mathrm{~kg} . \mathrm{m}^{-3}$ para uma mistura de todos os resíduos.

Não houve variação estatística entre os tratamentos analisados, e a menor granulometria da amostra 1 não afetou significativamente a densidade dos briquetes obtidos desta amostra em relação aos demais tratamentos.

O poder calorífico superior dos briquetes foi ligeiramente superior ao da matéria-prima. Para os briquetes ocorreu variação estatística entre os tratamentos na análise conjunta dos tratamentos e entre os briquetes feitos com falhas inteiras e moídas.

O poder calorífico líquido foi elevado em comparação com as falhas in natura. O ganho energético obtido com a compactação foi em função da redução do teor de umidade da matéria-prima (falhas) requerida para a compactação e consequentemente do produto final (briquetes). Este é um dos motivos que torna os compactados energeticamente mais eficientes que a matéria-prima que lhes dá origem. Somente o tratamento FM3, que teve o menor valor foi diferente dos demais tratamentos. Já a análise entre falhas inteiras e moídas também apresentou variação estatística com maior poder calorífico líquido para os briquetes de falhas inteiras.

Os teores de voláteis foram maiores para os briquetes em relação à matéria-prima e o carbono fixo menor. O teor de cinzas foi menor nos briquetes. Entre os briquetes não houve variação estatística entre os tratamentos para voláteis e carbono fixo, e para o teor de cinzas o tratamento FM1, com maior teor de cinzas, foi diferente de todos os demais tratamentos.

De forma geral, as propriedades dos briquetes de falhas inteiras e de falhas moídas foram similares, de modo que a qualidade dos briquetes não foi influenciada pelo tamanho das partículas.

\section{CONCLUSÕES}

- O teor de umidade das falhas in natura foi alto inviabilizando seu uso energético sem prévio tratamento. - Com base na composição química imediata e poder calorífico superior, comparativamente à madeira, as falhas de pinhão geram menor quantidade de energia por unidade de massa e maior quantidade de resíduos após a queima, no entanto queimam mais lentamente.

- As propriedades energéticas das falhas de pinhão são similares aos resíduos: bagaço de cana e ramas de algodão.

- A quantidade de lignina e extrativos das falhas de pinhão são maiores que na madeira, contribuindo para a boa ligação interna das partículas na produção de briquetes.

- Não é necessária a moagem das falhas para a produção de briquetes.

- A produção de briquetes a partir de falhas de pinhão pode ser uma alternativa viável de uso dos resíduos da cadeia produtiva do pinhão, gerando renda para pequenos produtores rurais. 
Jacinto et al. - Análise da qualidade energética da falha de pinhão para a produção de briquetes

\section{AGRADECIMENTOS}

As empresas Soluções e Inovações em Tecnologia de Madeiras; Tractebel Energia S.A.; Universidade do Planalto Catarinense; pela bolsa de iniciações científica da Universidade do Estado de Santa Catarina e ao Projeto de Pesquisa Kayuvá.

\section{REFERÊNCIAS BIBLIOGRÁFICAS}

ABNT - ASSOCIAÇÃO BRASILEIRA DE NORMAS TÉCNICAS. NBR 14577: Determinação do material solúvel em água. 2003a.

ABNT - ASSOCIAÇÃO BRASILEIRA DE NORMAS TÉCNICAS. NBR 14929: Madeira - Determinação do teor de umidade de cavacos - Método por secagem em estufa. 2003b.

ABNT - ASSOCIAÇÃO BRASILEIRA DE NORMAS TÉCNICAS. NBR 14853: Madeira - Determinação do material solúvel em etanol-tolueno e em diclorometano e em acetona. 2010a.

ABNT - ASSOCIAÇÃO BRASILEIRA DE NORMAS TÉCNICAS. NBR 7989: Pasta celulósica e madeira Determinação de lignina insolúvel em ácido. Rio de Janeiro, 2010b.

ASTM - AMERICAN SOCIETY FOR TESTING AND MATERIALS. D-1762: Standard Test Method for Chemical Analysis of wood charcoal. 2007.

BALLONI, C. J. V. Caracterização física e química da madeira de Pinus elliottii. 2009. 41 p. Monografia (Graduação em Engenharia Industrial Madeireira) - Universidade Estadual Paulista "Júlio de Mesquita Filho". Itapeva, 41 p.

BRAND, M. A. Energia de Biomassa florestal. Rio de Janeiro: Interciência, 2010. 114 p.

BRITO, J. O.; NUCCI, O. Estudo tecnológico da madeira de Pinus spp para a produção de carvão vegetal e briquetagem. IPEF, Piracicaba, n. 26, p. 25-30, 1984.

DIAS, J. M. C. S.; SOUZA, D. T.; BRAGA, M.; ONOYAMA, M. M.; MIRANDA, C. H. B.; BARBOSA, P. F. D.; ROCHA, D. J. Produção de briquetes e pellets a partir de resíduos agrícolas, agroindustriais e florestais. Brasília: Embrapa Agroenergia, 2012.

DIN. DEUTSCHES INSTITUT FÜR NORMUNG. DIN 51900: Determining the gross calorific value of solid and liquid fuels using the bomb calorimeter, and calculation of net calorific value. Berlim, 2000.

FRIEDLET, A.; PADOUVAS, E.; ROTTER, H.; VARMUZA, K. Prediction of heating values of biomass fuel from elemental composition. Analytica Chimica Acta. v. 544, n. 1-2, p. 191-198, 2005.

FURTADO, T. S.; FERREIRA, J. C.; BRAND, M. A.; MUÑIZ, G. I. B.; QUIRINO, W. F. Mapeamento da frequência de uso e características da biomassa florestal utilizada para geração de energia em Lages, SC. Ciência Florestal, Santa Maria, v. 22, n. 4, p. 795-802, 2012.

FURTADO, T. S.; VALIN, M.; BRAND, M. A.; BELLOTE, A. F. J. Variáveis do processo de briquetagem e qualidade de briquetes de biomassa florestal. Pesquisa Florestal Brasileira, Colombo, v. 30, n. 62, p. 101-106, 2010.

GUERRA, M. P.; SILVEIRA, V.; REIS, M. S.; SCHNEIDER, L. Exploração, manejo e conservação da Araucária (Araucaria angustifolia). In: SIMÕES, L. L.; LINO, C. F. (Org.). Sustentável Mata Atlântica: a exploração de seus recursos florestais. São Paulo: Editora SENAC São Paulo, 2002. p. 85-101.

KALIYAN, N.; MOREY, R. V. Factors affeting strength and durability of densified biomass products. Biomass and Bioenergy, Oxford, v. 33, n. 3, p. 337-359, 2009. 
MANTOVANI, A.; MORELlATO, L. P. C.; REIS, M. S. Fenologia reprodutiva e produção de sementes em Araucaria angustifolia (Bert.) O. Kuntze. Revista Brasileira de Botânica, São Paulo, v. 27, n. 4, p. 787-796, 2004.

MORAES, S. A. L.; NASCIMENTO, E. A.; MELO, D. C. O. Análise da madeira de Pinus oocarpa parte I - estudo dos constituintes macromoleculares e extrativos voláteis. Revista Árvore, Viçosa, v. 29, n. 3, p. 461-470, 2005.

OBERNBERGER, I.; THEK, G. Physical characterisation and chemical composition of densified biomass fuels with regard to their composition behaviour. In: WORLD CONFERENCE ON PELLETS, 1. Proceedings... Estocolmo, 2002, p. 115-121.

OLIVEIRA, J.; LOPES, G. P. R. D.; BRUTTI, R. C. Caracterização energética da "falha do pinhão". In: SIMPÓSIO DE PÓS-GRADUAÇÃO EM CIÊNCIAS FLORESTAIS, 6; SIMPÓSIO DE CIÊNCIA E TECNOLOGIA DA MADEIRA DO RJ, 2, 2010, Rio de Janeiro. Anais... Rio de Janeiro: Instituto de Florestas, 2010.

PARIKH, J.; CHANNIWALA, S. A.; GHOSAL, G. K. A correlation for calculating HHV from proximate analysis of solid fuels. Fuel, v. 84, n. 5, p. 487-494, 2005.

PROTÁSIO, T. P.; ALVES, I. C. N.; TRUGILHO, P. F.; SILVA, V. O.; BALIZA, A. E. R. Compactação de biomassa vegetal visando à produção de biocombustíveis sólidos. Pesquisa Florestal Brasileira, Colombo, v. 31, n. 68, p. 273-283, 2011.

SANTOS, S. C. Índios e Brancos no Sul do Brasil: a dramática experiência dos Xokleng. Florianópolis: Ed. Edune, 1973. 312 p.

VIEIRA, E. E. Simbolismo e reelaboração na cultura material dos Xokleng. 2004. 122 p. Dissertação (Mestrado em História) - Universidade Federal de Santa Catarina, Florianópolis, 2004.

VIEIRA-DA-SILVA, C.; MIGUEL, L. A.; REIS, M. S. Utilizações alternativas para a "falha", componente da pinha (Araucaria angustifolia), e seu potencial para a agricultura de base agroecológica. Cadernos de Agroecologia, v. 6, n. 2, p. 1-5, 2011.

VIEIRA-DA-SILVA, C.; REIS, M. S. Produção de pinhão na região de Caçador, SC: aspectos da obtenção e sua Importância para comunidades locais. Ciência Florestal. Santa Maria, v. 19, n. 4, p. 363-374, 2009.

WERTHER, J.; SAENGER, M.; HARTGE, E. U.; OGADA, T.; SIAGI, Z. Combustion of agricultural residues. Progress in Energy and Combustion Science. Hamburg, v. 26, n. 1, p. 1-27, fev. 2000.

Recebido em 26/03/2015

Aceito para publicação em 18/04/2016

Sci. For., Piracicaba, v. 44, n. 112, p. 821-829, dez. 2016 DOI: dx.doi.org/10.18671/scifor.v44n112.04 
